\title{
EVENTOS ADVERSOS IDENTIFICADOS NOS RELATÓRIOS DE ENFERMAGEM EM UMA CLÍNICA PEDIÁTRICA
}

\author{
ADVERSE EVENTS IDENTIFIED IN NURSING REPORTS IN \\ A PEDIATRIC CLINICAL
}

\section{EVENTOS ADVERSOS IDENTIFICADOS EN LOS INFORMES DE ENFERMERÍA EN UNA CLÍNICA PEDIÁTRICA}

\author{
Judite Pereira Rocha* \\ Ana Elisa Bauer de Camargo Silva ${ }^{* *}$ \\ Ana Lúcia QueIroz Bezerra ${ }^{* * *}$ \\ Maiana Regina Gomes de Sousa ${ }^{* * * *}$ \\ ISADORA Alves MOREIRA ${ }^{* * * * *}$
}

\begin{abstract}
RESUMO
Objetivo: Identificar os eventos adversos ocorridos na unidade de pediatria de um hospital de ensino de Goiânia-Goiás-Brasil. Método: Pesquisa descritiva, retrospectiva e documental, com abordagem quantitativa. A coleta foi realizada em dois períodos: outubro de 2011 a fevereiro de 2012 e em agosto de 2014 . Os dados foram obtidos de relatórios de enfermagem preenchidos no período de junho de 2006 a junho de 2013. A análise estatística foi realizada no programa SPSS, versão 19.0 for Windows. Resultados: Foram identificados 556 eventos adversos ocorridos durante a assistência na clínica Pediátrica, sendo que os mais prevalentes foram os relacionados ao acesso vascular (40,8\%), seguido dos relacionados a sondas, cateteres, drenos e tubos $(27,2 \%)$ e a medicamentos (15,5\%). Conclusão: Considera-se que os resultados deste estudo possibilitem uma análise dos riscos a que as crianças estão expostas e o planejamento de ações a serem trabalhadas para a melhoria no processo de cuidado.
\end{abstract}

Palavras chave: Enfermagem, enfermagem pediátrica, segurança do paciente.

\begin{abstract}
Descriptive, retrospective and documentary research with quantitative approach that aims to identify adverse events occurred in the pediatric unit of a teaching hospital in Goiânia, Goiás, Brazil. Data collection was conducted in two periods: October 2011 to February 2012 and August 2014, and data were obtained from reports of nursing. Completed reports filled from June 2006 to June 2013 were analyzed. The data were structured at a Microsoft Excel spreadsheets version 2010 and statistically analyzed using the SPSS software version 19.0. There

\footnotetext{
* Enfermeira. Graduada pela Faculdade de Enfermagem da Universidade Federal de Goiás. Participante do Programa Institucional de Bolsas de Iniciação Científica. Goiânia, Goiás, Brasil. E-mail: juditrocha@hotmail.com

${ }^{* *}$ Enfermeira. Doutora em Enfermagem. Professora Adjunta da Faculdade de Enfermagem da Universidade Federal de Goiás. Goiânia, Goiás, Brasil. E-mail: anaelisa@terra.com.br. Telefone: 55 (62) 3209-6280 - Fax: 55 (62) 3209-6282

${ }^{* * *}$ Enfermeira. Doutora em Enfermagem. Professora Associada da Faculdade de Enfermagem da Universidade Federal de Goiás. Goiânia, Goiás, Brasil. E-mail: analuciaqueiroz@uol.com.br

${ }^{* * * *}$ Enfermeira. Mestranda do Programa de Pós-Graduação da Faculdade de Enfermagem da Universidade Federal de Goiás. Goiânia, Goiás, Brasil. E-mail: maianaregina@gmail.com

${ }_{* * * * *}^{*}$ Enfermeira. Residente da área de Urgência e Emergência no Programa de Residência Multiprofissional do Hospital das Clínicas da Universidade Federal de Goiás. Goiânia, Goiás, Brasil. E-mail: isadora.a.moreira@gmail.com
} 
were 556 adverse events identified that occurred during pediatric clinical assistance, and the most prevalent ones were related to vascular access $(40.8 \%)$, followed by the related probes, catheters, drains and pipes (27.2\%), and drug-related (15.5\%). It is considered that the results of this study allow an analysis of the risks to which children are exposed and planning actions to be worked for the improvement in the care process.

Key words: Nursing, pediatric nursing, patient safety.

\section{RESUMEN}

Investigación descriptiva, retrospectiva y documental con enfoque cuantitativo, que tuvo como objetivo identificar los efectos adversos ocurridos en una unidad pediátrica de un hospital universitario de Goiânia, Goiás, Brasil. La recolección de datos se realizó en dos períodos: octubre 2011 a febrero 2012 y agosto de 2014, y los datos fueron obtenidos de los registros de enfermería. Fueron analizados los registros llenados entre junio 2006 y junio 2013. Los datos fueron estructurados en el software Microsoft Excel versión 2010 y analizados estadísticamente usando el software SPSS versión 19.0. Fueron identificados 556 eventos adversos durante la asistencia clínica-pediátrica, y los más frecuentes eran relacionados con el acceso vascular (40,8\%), seguido por las relacionadas a las sondas, catéteres, drenajes y tuberías (27,2\%), seguidos por los relacionados con las drogas $(15,5 \%)$. Se considera que los resultados de este estudio permiten un análisis de los riesgos a que están expuestos los niños y la planificación de acciones que pueden ser hechas para la mejora en el proceso de atención.

Palabras clave: Enfermería, enfermería pediátrica, seguridad del paciente.

Fecha recepción: 13/10/13. Fecha acepctación: 17/07/14.

\section{INTRODUÇÃO}

Atualmente as consequências dos incidentes ocorridos durante a assistência em saúde têm ganhado espaço na mídia televisiva e escrita. Os danos causados por incidentes na prestação do cuidado à saúde são denominados Eventos Adversos (EA), e podem ser de natureza física, social ou psicológica (1). A ocorrência desses EA evidencia a qualidade da assistência prestada (2).

Os EA podem acontecer em diversos serviços de uma instituição hospitalar, incluindo as unidades de pediatria, nas quais a ocorrência desses eventos é comum devido às características peculiares de uma criança, além das lacunas deixadas pela presença de vários atores envolvidos no cuidado à criança hospitalizada, tais como equipe multiprofissional, pais e/ou acompanhantes (3-4).

Os EA em crianças podem ser mais graves do que em adultos, devido à imaturidade funcional, que as deixam mais vulneráveis aos danos, e por haver maior dificuldade na detecção do evento nesse grupo. As crianças diferem dos adultos em muitos aspectos no que diz respeito à assistência médica, portanto, é provável que a epidemiologia dos EA em crianças também seja significativamente diferente (3-5).

Estudo realizado em hospitais americanos mostrou que os EA ocorreram em 1\% das hospitalizações pediátricas, sugerindo que aproximadamente 70.000 crianças sofrem um EA a cada ano (5).

No Brasil, em uma unidade de cuidados intensivos pediátricos de um hospital universitário de São Paulo, foram identificados, em três meses, 113 EA de vários tipos, sendo que os eventos mais frequentes foram relacionados à medicação (32,7\%), seguido de eventos relacionados à ventilação pulmonar mecânica/via aérea (29,2\%), cateteres/sondas e drenos $(14,13 \%)$ e eventos relacionados a procedimentos de enfermagem (16,8\%). Dentro dessa última categoria, destacaram-se a não execução da prescrição de enfermagem, a uti- 
lização de curativo inadequado ao tratamento, à execução incorreta de técnica e omissão do cuidado (6). Esses eventos acarretam preocupação tanto aos profissionais como aos acompanhantes das crianças, sendo os erros de medicação os mais prevalentes (7).

Estudo que avalia a prescrição de medicamentos em pediatria afirma que a frequência de erros envolvendo medicação em pacientes pediátricos chega a representar 1 para cada 6,4 prescrições, muitas vezes sendo erros de intensidade grave, ou seja, três vezes maior que a frequência relacionada em adultos (8).

A equipe de saúde, principalmente a enfermagem, por ser uma das categorias com maior força de trabalho, deve ter competências para analisar os fatores de risco a que as crianças podem estar expostas, com o objetivo de prevenir falhas durante a assistência, assegurando um cuidado livre de $\operatorname{EA}(4,9)$.

Neste contexto, este estudo se propõe a realizar um levantamento dos principais EA relacionados à criança, fornecendo assim informações para o planejamento de estratégias de melhorias que aumentem a segurança do paciente pediátrico, além de despertar o interesse dos profissionais e pesquisadores para a realização de mais pesquisas nessa área, com foco na busca por maior qualidade no atendimento à criança. Reconhecer e identificar os problemas envolvendo o cuidado pediátrico é considerado umas das primeiras ações para desenvolver uma cultura de segurança na assistência a essa categoria (10).

Sendo assim, esse estudo teve como objetivo identificar os eventos adversos ocorridos na unidade de pediatria de um hospital ensino de Goiânia-Goiás-Brasil, além de classificá-los quanto ao tipo e frequência.

\section{MATERIAL E MÉTODO}

Estudo descritivo, retrospectivo e documental, com abordagem quantitativa, desenvolvido na unidade de pediatria de um hospi- tal ensino de Goiânia-Goiás-Brasil, que faz parte da Rede Hospitais Sentinela da Agência Nacional de Vigilância Sanitária.

A unidade de Pediatria atende crianças que já são acompanhadas no serviço ambulatorial do hospital para tratamento clínico periódico, ou aquelas provindas do Serviço de Urgências Pediátricas e da Unidade de Terapia Intensiva Neonatal do próprio hospital. As crianças são alojadas nos 27 leitos, distribuídos em cinco enfermarias, de acordo com a especialidade clínica, sendo cinco leitos para nefrologia, seis para clínica geral, cinco para gastrologia, seis para cirurgias pediátricas, dois para reumatologia, dois para pneumologia e um para isolamento.

A equipe de enfermagem, durante a pesquisa, estava composta por oito enfermeiros, sendo sete assistencialistas e um coordenador da unidade, dezesseis técnicos de enfermagem, seis auxiliares de enfermagem e dois bolsistas estudantes de enfermagem. Os profissionais da unidade cumpriam carga horária de $30 \mathrm{~h}$ semanais, com exceção do coordenador que realizava $40 \mathrm{~h}$ semanais.

Para a obtenção dos dados foi realizada a leitura do conteúdo dos livros utilizados pela enfermagem, principalmente enfermeiros, no período de junho de 2006 a junho de 2013, para registros de informações sobre as ocorrências do plantão, intercorrências, avisos, comunicados internos e as solicitações realizadas. Analisou-se o conteúdo das anotações, visando identificar os registros relacionados à ocorrência de EA. A coleta dos dados foi realizada no período de outubro de 2011 a fevereiro de 2012 e em agosto de 2014. As anotações encontradas foram inseridas em um instrumento de coleta de dados, validado por três especialistas, contendo à transcrição da anotação tal qual redigida e o tipo de EA.

A população do estudo foi constituída por 556 eventos adversos, descritos nos relatórios de enfermagem no período em análise.

Os dados foram estruturados em planilhas eletrônicas do Microsoft Excel versão 2007 e 
a análise estatística foi realizada no programa SPSS, versão 19.0 for Windows.

Estudo vinculado ao projeto Análise de ocorrências de eventos adversos em um hospital da rede sentinela na região Centro Oeste, o qual foi submetido à apreciação pelo Comitê de Ética em Pesquisa Médica Humana e Animal do Hospital das Clínicas da Universidade Federal de Goiás, recebendo parecer favorável (Protocolo No 064/2008). Todos os critérios foram atendidos conforme a Resolução No 196/96 e 466/12 do Conselho Nacional de Saúde (11).

\section{RESULTADOS}

A análise dos dados permitiu caracterizar os 556 eventos adversos identificados por tipo de evento, segundo ano de ocorrência e frequência. A Tabela 1 demonstra os tipos de EA encontrados, assim como as frequências absolutas e relativas.

Os eventos relacionados ao acesso vascular foram os mais prevalentes com um quantitativo de 227 (40,8\%), por este motivo eles serão descritos de forma mais detalhada na Tabela 2.

Tabela 1. Distribuição dos eventos adversos identificados na clínica pediátrica por tipo de evento e frequência, no período de 2006-2013. Goiânia, Goiás, Brasil, 2014.

\begin{tabular}{lrrrrrrrrrrr}
\hline & \multicolumn{4}{c}{ Ano } & \multicolumn{4}{c}{ Total } \\
\cline { 2 - 10 } Tipo de evento & 2006 & 2007 & 2008 & 2009 & 2010 & 2011 & 2012 & 2013 & N & $\%$ \\
\hline Relacionados ao acesso vascular & 15 & 36 & 27 & 46 & 35 & 38 & 20 & 10 & 227 & $40,8 \%$ \\
Relacionados a sondas, cateteres, drenos, e tubos & 6 & 23 & 23 & 20 & 23 & 24 & 21 & 11 & 151 & $27,2 \%$ \\
Relacionados a medicamentos & 8 & 11 & 13 & 4 & 17 & 18 & 13 & 2 & 86 & $15,5 \%$ \\
Sinais de infecção em feridas & 0 & 2 & 0 & 2 & 1 & 8 & 5 & 1 & 19 & $3,4 \%$ \\
Evasão & 0 & 0 & 2 & 2 & 3 & 1 & 5 & 3 & 16 & $2,9 \%$ \\
Infecção & 0 & 0 & 5 & 1 & 0 & 2 & 7 & 0 & 15 & $2,7 \%$ \\
Queda & 1 & 2 & 1 & 2 & 2 & 2 & 1 & 0 & 11 & $2,0 \%$ \\
Úlcera por pressão & 1 & 1 & 0 & 0 & 2 & 0 & 1 & 0 & 5 & $0,9 \%$ \\
Processos alérgicos & 0 & 1 & 0 & 0 & 0 & 2 & 1 & 1 & 5 & $0,9 \%$ \\
Outros & 0 & 1 & 2 & 2 & 13 & 3 & 0 & 0 & 21 & $3,8 \%$ \\
\hline Total & 31 & 77 & 73 & 79 & 96 & 98 & 74 & 28 & 556 & $100,0 \%$ \\
\hline
\end{tabular}

Tabela 2. Distribuição dos eventos adversos relacionados ao acesso vascular, identificados na clínica pediátrica, segundo tipo de evento e frequência, no período de 2006-2013. Goiânia, Goiás, Brasil, 2014.

\begin{tabular}{lrr}
\hline Tipo de evento & $\mathbf{N}$ & $\%$ \\
\hline Insucesso na punção venosa & 114 & $51,1 \%$ \\
Retirada não programada & 35 & $15,7 \%$ \\
Obstrução & 35 & $15,7 \%$ \\
Sangramento & 18 & $8,1 \%$ \\
Sinais flogísticos & 17 & $7,6 \%$ \\
Cateter ineficaz & 3 & $1,3 \%$ \\
Extravasamento & 1 & $0,4 \%$ \\
\hline Total & $\mathbf{2 2 3}$ & $\mathbf{1 0 0 , 0} \%$ \\
\hline
\end{tabular}


Os eventos caracterizados com insucesso na punção venosa englobam punções sem sucesso e as que só obtiveram sucesso após exaustivas tentativas. Quanto aos eventos que relacionados à retirada não programada do acesso vascular, 20 (57,1\%) estavam relacionados a acesso vascular periférico e 15 $(42,9 \%)$ a acesso vascular central.

Todos os eventos de sangramento estavam relacionados à incisão do cateter venoso, sendo haver alguns relatos em que os sangramentos eram em forma de "jato", sangramento vivo e contínuo.

Nos eventos do tipo sinais flogísticos foram observadas a presença de hiperemia, edema e secreção purulenta. Quanto a cateter ineficaz, os eventos foram em decorrência do dobramento e rachadura no mesmo.

A distribuição dos 151 eventos relacionados a sondas, cateteres, drenos e tubos, também muito prevalentes, será apresentada na Tabela 3.

Tabela 3. Distribuição dos eventos adversos relacionados a sondas, cateteres, drenos, e tubos na clínica pediátrica, quanto ao tipo e frequência, no período de 2006-2013. Goiânia, Goiás, Brasil, 2014.

\begin{tabular}{llrcc}
\hline \multicolumn{1}{c}{ Tipo de evento } & N & \% (ESPECIFICO) & \% (TOTAL) \\
\hline Relacionados a sonda: & Nasogástrica & 40 & $30,3 \%$ & $26,5 \%$ \\
& Nasoentérica & 33 & $25,0 \%$ & $21,9 \%$ \\
& Orogátrica & 16 & $12,1 \%$ & $10,6 \%$ \\
& Vesical & 14 & $10,6 \%$ & $9,3 \%$ \\
& Gastrostomia & 12 & $9,1 \%$ & $7,9 \%$ \\
& pHmetria & 4 & $3,0 \%$ & $2,6 \%$ \\
& Uretral & 2 & $1,5 \%$ & $1,3 \%$ \\
& Retal & 1 & $0,8 \%$ & $0,7 \%$ \\
& Oxigênia & 1 & $0,8 \%$ & $0,7 \%$ \\
& Cistostomia & 1 & $0,8 \%$ & $0,7 \%$ \\
& Não especificado & 8 & $6,1 \%$ & $5,3 \%$ \\
\cline { 2 - 5 } & Subtotal (sonda) & 132 & $100,0 \%$ & $87,4 \%$ \\
\hline Relacionados a drenos & & 10 & $100,0 \%$ & $6,6 \%$ \\
\hline \multirow{2}{*}{ Relacionados a cateteres: } & Abdominal & 2 & $28,6 \%$ & $1,3 \%$ \\
& FAV & 2 & $28,6 \%$ & $2,3 \%$ \\
& Não especificado & 3 & $42,9 \%$ & $4,6 \%$ \\
\cline { 2 - 5 } & Subtotal (cateteres) & 7 & $100,0 \%$ & $0,7 \%$ \\
\hline Relacionados a cânulas: & Traqueostomia & 1 & $50,0 \%$ & $0,7 \%$ \\
\hline & Entubação & 1 & $50,0 \%$ & $1,3 \%$ \\
\cline { 2 - 5 } & Subtotal(cânula) & 2 & $100,0 \%$ & $100,0 \%$ \\
\hline & Total & 151 & $100,0 \%$ & \\
\hline
\end{tabular}

Dentre os 40 eventos envolvendo sondas nasogástricas, $30(75 \%)$ foram por retirada não programada da sonda, seis $(15 \%)$ por obstrução da sonda e quatro (10\%) devido a insucesso/dificuldade na passagem da sonda.
Nos eventos relacionados às sondas nasoentéricas, $21(63,6 \%)$ foram por saída/retirada acidental da sonda, $11(33,3 \%)$ por obstrução da sonda e um (3\%) por insucesso/ dificuldade na passagem. 
Entre os 16 eventos relacionados à sonda orogástrica, $15(93,8 \%)$ foram por retirada não programada e um $(6,3 \%)$ devido obstrução da sonda.

Dos 14 eventos que se referiam à sonda vesical, seis $(42,2 \%)$ foram por retirada não programada da sonda, cinco $(35,7 \%)$ por insucesso/dificuldade na passagem, dois $(14,3 \%)$ por ineficiência da sonda, devido à presença de rachadura na mesma, e um $(7,1 \%)$ por sangramento no momento do procedimento de passagem da sonda.

Com relação aos eventos ocorridos com sonda de gastrostomia, sete $(58,3 \%)$ foram por retirada não programada da sonda, quatro $(33,3 \%)$ devido à presença de sinais de infecções e um $(8,3 \%)$ por ineficiência da sonda, por apresentar furos em sua extensão.

Entre os eventos relacionados à sonda de pHmetria, sonda uretral, sonda retal e sonda de oxigênio, todos ocorreram por retirada não programada das sondas. O único evento relacionado à sonda de cistostomia ocorreu por ineficiência da sonda, com vazamento de urina na inserção.

Por fim, em relação aos oito eventos ocorridos sem especificação do tipo de sonda, quatro $(40 \%)$ foram por retirada não programada da sonda, um (12,5\%) por insucesso/dificuldade na passagem, um (12,5\%) por obstrução, um $(12,5 \%)$ por sangramento contínuo na inserção da sonda e um $(12,5 \%)$ por sonda ineficaz, devido à presença de rachadura.

Os eventos que se referiram aos cateteres sem especificação foram por retirada não programada. Os eventos relacionados a cateter abdominal foram devido à presença de sangramento e retirada não programada. $\mathrm{O}$ único evento relacionado à fístula arteriovenosa foi por sangramento significativo.

Com relação aos drenos, os EA ocorreram em cinco $(50 \%)$ casos por retirada não programada do dreno, dois (20\%) foram referentes a sinais de infecção, dois (20\%) por sangramento e um (10\%) foi por obstrução.

Os eventos relacionados à cânula de tra- queostomia e intubação foram por retirada não programada da cânula.

No concernente aos eventos relacionados aos medicamentos, foram identificados 86 EA, sendo 27 (31,4\%) erros de omissão, 27 $(31,4 \%)$ processos alérgicos, $19(22,1 \%)$ reações que não foram especificadas as causas, oito $(9,3 \%)$ erros de horário e dois $(2,3 \%)$ administrações de medicamento não prescrito.

Constataram-se ainda 19 ocorrências com sinais de infecção em feridas, porém sem o diagnóstico, apresentando secreção purulenta, secreção esverdeada, odor forte e hiperemia. Nos casos de infecção, quatro (26,7\%) foram por Pseudomonas, três (20\%) Scherichia coli, três (20\%) Staphylococcus aureus resistente à meticilina, dois $(13,3 \%)$ Klebisiella, dois $(13,3 \%)$ por sepse, um $(6,7 \%)$ por Herpes zoster.

No concernente aos eventos de evasão, em todos os casos a mãe evadiu-se levando a criança e interrompendo o tratamento. Quanto aos eventos tipo queda, três $(27,3 \%)$ foram da cama, três $(27,3 \%)$ do berço, duas $(18,2 \%)$ da própria altura, e as demais foram queda da maca $(9,1 \%)$, do balanço $(9,1 \%)$ e local não especificado $(9,1 \%)$.

Em relação 5 às úlceras por pressão, 2 ocorreram na região sacral, uma no glúteo esquerdo, uma na região lombosacral e um caso sem registro do local. Quanto aos processos alérgicos, um caso se referia ao uso de esparadrapo e os demais não foram especificadas as causas, tendo como sintomas prurido por todo o corpo.

Houve 21 eventos que não foram enquadrados dentro das categorias, sendo classificados como "outros tipos". Desses, 13 $(61,9 \%)$ se referiam a exames cancelados, dois $(9,5 \%)$ relacionados à troca de água por álcool, um $(4,8 \%)$ a insucesso na punção suprapúbica, um $(4,8 \%)$ a cirurgia cancelada, um $(4,8 \%)$ a hipoglicemia, um $(4,8 \%)$ por uso inadequado de oxigênio, um $(4,8 \%)$ sangramento intenso na punção arterial (artéria femoral) e um $(4,8 \%)$ por auto-medicação 
- a mãe da criança tinha várias medicações dentro da bolsa e administrava na criança sem autorização dos profissionais.

No evento relacionado à hipoglicemia a criança ficou cinco horas de dieta zero para punção central na sala de operação, e houve redução da glicemia sendo necessária a administração de glicose; em relação ao evento sobre uso inadequado de oxigênio a prescrição era de $2 \mathrm{~L} / \mathrm{min}$ de oxigênio, porém a acompanhante da criança colocou menos de $1 \mathrm{~L} / \mathrm{min}$, mesmo após várias explicações da enfermagem sobre a importância de manter a terapia de acordo com a prescrita. No evento identificado como troca de água por álcool foi oferecido álcool ao paciente no lugar de água: "A mãe do menor deixou a mamadeira cheia de álcool em cima do criado, a avó chegou para troca de acompanhante e ofereceu a mamadeira para a criança achando que era água". E em outro caso: "ao invés de infundir água filtrada por sonda, infundiu álcool 70\%. O frasco do álcool e o que é usado para dieta estavam na mesa ao lado do berço da criança, sem identificação. Mãe refere que colocou o frasco achando que era água".

\section{DISCUSSÃO E CONCLUSÃO}

Os resultados deste estudo mostraram o potencial dos registros de enfermagem como fonte de informações sobre EA. Com estas informações é possível fazer um diagnóstico situacional, pois foi identificada a ocorrência de muitos e variados tipos de EA na unidade de pediatria, durante o período analisado.

Neste estudo, os EA identificados foram predominantemente os relacionados a acesso vascular e, dentre eles, prevaleceu o insucesso/dificuldade durante o procedimento de punção venosa.

É importante destacar que a punção venosa é uma prática rotineira nas unidades de pediatria devido ao fato da terapia intravenosa ser um dos procedimentos mais realiza- dos durante a hospitalização.

No entanto, para realização deste procedimento com eficácia e efetividade é necessário conhecimento de anatomia, fisiologia da pele e do sistema venoso, além de preparar a criança emocionalmente, informar sobre o procedimento a ser realizado e valorizar a presença de seu acompanhante. Todas essas variáveis tem que estar presentes para minimizar a dor, medo e o sofrimento nas crianças que podem ser submetidas a exaustivas tentativas de punções venosas, o que aumenta o risco para um trauma vascular periférico $(12,13)$.

As crianças internadas estão susceptíveis a inúmeros procedimentos dolorosos, portanto, cabe à enfermagem minimizar as situações de sofrimento da criança, com ações ponderadas sobre o momento a realizar o procedimento; uso de técnica com perícia e delicadeza; a escolha criteriosa quanto ao material a ser utilizado (flexibilidade, tamanho); uso de medidas não farmacológicas, a aplicação de anestésicos locais e envolver os pais nos cuidados prestados (14).

A tecnologia também pode contribuir com essa prática, como exemplo, temos a utilização da ultra-sonografia para guiar a punção venosa reduzindo assim, as várias tentativas de punção em um mesmo paciente (15).

Os eventos relacionados a cateteres, sondas, drenos e tubos tem sido identificados em vários estudos. Em Goiás, um estudo listou $162(61,36 \%)$ EA relacionados a tais artefatos terapêuticos (16). Em São Paulo, outro estudo realizado em uma unidade de cuidados intensivos pediátricos evidenciou que os EA com dispositivos tubulares corresponderam a $14,3 \%$ do total de 113 ocorrências investigadas (6).

A maioria dos eventos com esses dispositivos foi por retirada não programada, o que implica na necessidade de maior atenção por parte dos profissionais com a manipulação desses artefatos, monitoração dos pacientes e orientações ao acompanhante e à própria criança. 
A retirada não programada desses dispositivos tem como consequência um aumento do tempo e custo direcionado a assistência do paciente uma vez que muitas vezes é necessário a reimplantação desse cateter, sonda e/ou dreno (17).

A prevalência de EA relacionados a erros de omissão é reafirmada por outro estudo realizado em São Paulo, no qual foram registradas 71 notificações de 110 erros de medicação em 92 dias, e destes o erro de omissão foi o mais frequente $(22,7 \%)$ (18).

Cabe ressaltar que dentre esses medicamentos que não foram administrados estão vários antibióticos. Os erros de omissão de antibióticos são falhas que devem ser evitadas, pois a omissão de uma dose pode comprometer a eficácia de todo o tratamento, devido às modificações do seu nível sérico (19, 20).

Uma das estratégias para prevenir erros de omissão de medicamentos é instituir meios eficazes de comunicação entre a equipe multiprofissional, entre os integrantes da equipe e o paciente e família (21) para reduzir o risco da não administração do medicamento prescrito ao paciente.

Os EA envolvendo queda no ambiente hospitalar, também presentes neste estudo, são outro tipo de intercorrência considerado comum no contexto da segurança do paciente (22) e isso deve ser um sinal de alerta, uma vez que pode trazer complicações à integridade física e emocional dos pacientes, além das consequências econômicas para a instituição (23). Um estudo realizado em prontuários de pacientes, que apresentaram quedas em um hospital nos Estados Unidos, identificou que $82 \%$ das crianças estavam acompanhadas dos pais durante as quedas (24).

Outro estudo identificou que crianças com menos de cinco anos de idade e do sexo masculino caíram com mais frequência, sendo que a maior parte das quedas ocorreu no quarto da criança $(32,4 \%)$, seguida pelo banheiro $(29,6 \%)$. Das 71 quedas relatadas, $45 \%$ sofreram uma lesão documentada (25).
Os conhecimentos sobre as quedas reforçam a adoção de medidas de prevenção como a elevação de grades nas camas, aplicação de técnicas seguras de restrição no leito, acompanhamento rigoroso da enfermagem, existencia de protocolos para prevenção de quedas e a participação da família da criança na identificação e prevenção desse tipo de evento. O desenvolvimento de um instrumento de avaliação de risco para identificar as crianças internadas com risco de quedas também é válido e muito importante (25).

Os eventos de evasão colaboram para reafirmar a necessidade de que a equipe multiprofissional deve ficar em constante vigilância aos pacientes e acompanhantes da criança hospitalizada, pois, a instituição de saúde e seus profissionais são responsáveis juridicamente por esse tipo de evento (26-27).

Os eventos relacionados à feridas com sinais de infecção e infecção propriamente dita apresentaram um quantitativo de $3,4 \%$ e $2,7 \%$ ocorrências respectivamente. As infecções são um problema de saúde pública podendo ser causadas por microrganismos pertencentes à própria microbiota do paciente ou transmitidas pelas mãos dos profissionais de saúde ou outras pessoas que entrem em contato com o paciente (28).

O primeiro Desafio Global para Segurança do Paciente da Aliança Mundial para Segurança do Paciente foi sobre o tema "Uma assistência segura, é uma assistência limpa" que visa o desenvolvimento de ações para reduzir os casos de infecções relacionadas à assistência à saúde (IRAS) em todo o mundo, pois estão entre as principais causas de morte em pacientes hospitalizados (29).

As consequências das IRAS implicam em prejuízos tanto para a instituição quanto para o paciente, como hospitalização prolongada, aumento da resistência de microrganismos aos agentes antimicrobianos e elevação dos custos financeiros. Portanto, IRAS é um grande problema para a segurança do paciente e sua vigilância e prevenção deve ser uma prioridade para as instituições para ga- 
rantir um cuidado mais seguro (30).

A higienização das mãos é a medida mais prática e relevante para evitar a transmissão de microrganismos (31), portanto, é fundamental que os profissionais de saúde tenham consciência da importância desse ato e adotem essa prática para reduzir a ocorrência de IRAS, garantindo a qualidade do cuidado.

Apesar de terem sido identificados apenas cinco casos de úlcera por pressão acredita-se que este tipo de EA deve ser analisado com cautela pela equipe de enfermagem uma vez que essas ocorrências prolongam a hospitalização, dificultam a recuperação do paciente ao aumentar o risco de complicações, elevam os custos do tratamento com recursos humanos e materiais além de representarem uma piora no sofrimento físico como emocional dos clientes (32).

No presente estudo, as úlceras por pressão ocorreram nas regiões sacral, glúteo esquerdo e lombosacral, diferindo de um estudo realizado em unidade de terapia intensiva pediátrica, no qual foi constatado que a região mais frequente de úlcera por pressão em crianças era a cabeça, principalmente na região occipital (33). Mesmo tendo tais divergências, o que destaca é que esse tipo de evento pode ser prevenido a partir de medidas simples como monitoramento, inspeção e proteção da pele, além de mudança de decúbito do paciente de $2 / 2 \mathrm{~h}$ (34).

O conhecimento dos tipos de EA prevalentes na unidade de pediatria é fundamental para analisar os riscos em que as crianças estão expostas e planejar as prioridades a serem trabalhadas para a melhoria no processo de cuidado.

Para realizar uma assistência de qualidade, faz-se necessário que os processos de trabalho sejam revisados, os profissionais sejam capacitados e treinados, sendo importante também que a instituição disponibilize tecnologias que possam ajudar nesse aprimoramento.

Um estudo também realizado em hospital escola em Goiás que avalia o cuidado pela equipe de enfermagem reforça a ideia que para oferecer um serviço em saúde com qualidade é importante estabelecer ações com bases científicas e instituir indicadores que permitam a avaliação do cuidado prestado além de fortalecer o envolvimento e trabalho em equipe (35).

Outro fator importante é que haja uma cultura de notificação nas instituições, para que as informações sobre falhas ou eventos adversos sejam claras e completas, promovendo uma melhor análise das suas causas. Ressalta-se que, como os relatórios de enfermagem são uma fonte de informações, porém não sistematizada, de ocorrências de EA, muitos registros encontrados estavam incompletos, o que dificultou a análise dos eventos e de suas causas.

Considera-se que os resultados do estudo possibilitem uma maior reflexão sobre a necessidade de que medidas de prevenção de eventos adversos sejam adotadas, minimizando complicações e sofrimento às crianças hospitalizadas. A prevenção de tais eventos exige esforços conjuntos de gestores, profissionais de saúde e pais para garantir a segurança do paciente pediátrico e a qualidade na assistência prestada.

\section{REFERÊNCIAS}

1. World Health Organization. The conceptual framework for the international classification for pacient safety [Internet]. En: Taxonomy. Geneva: WHO; 2007 [citado 01 agosto 2014]. Disponível em: http://www.who.int/patientsafety/taxonomy/en/.

2. Beccaria LM, Pereira RAM, Contrin LM, Lobo SMA, Trajano DHL. Eventos adversos na assistência de enfermagem em uma unidade de terapia intensiva. Rev Bras Ter Intensiva. 2009; 21(3).

3. Leal E, Brito MJ, Gouveia C, Varandas L, Ferreira GC. Iatrogenia em Pediatria: 
repensar atitudes. Acta Med Port. 2011; 24(2): 375-378.

4. Wegner W. A segurança do paciente nas circunstâncias de cuidado: prevenção de eventos adversos na hospitalização infantil. [Tese Doutorado]. Porto Alegre: UFRGS. Escola de Enfermagem da Universidade Federal do Rio Grande do Sul; 2011.

5. Woods D, Thomas E, Holl J, Altman S, Brennan T. Adverse events and preventable adverse events in children. Pediatrics. 2005; 115(1): 155-60.

6. Harada MJCS, Marin HF, Carvalho WB. Ocorrências Adversas e consequências Imediatas para os Pacientes em Unidade de Cuidados Intensivos Pediátricos. Acta Paul Enferm. 2003; 16(13): 62-70.

7. Wegner W, Pedro ENR. A segurança do paciente nas circunstâncias de cuidado: prevenção de eventos adversos na hospitalização infantil. Rev Lat Am Enfermagem. 2012; 20(3): 427-434.

8. Magalhães TC, Ferrari CKB, David FL. Aspectos críticos da prescrição de medicamentos em pediatria. Evidência - Ciência e Biotecnologia. 2013; 13(1): 5-18.

9. Conselho Federal de Enfermagem. Resolução COFEN No 311/2007. Aprova o Código de Ética dos Profissionais de Enfermagem. En: Conselho Regional de Enfermagem do Rio de Janeiro (COREN-RJ). Documentos básicos de enfermagem: enfermeiros, técnicos e auxiliares. Rio de Janeiro. COREN; 2007.

10. Santos JV, Santos, KPB, Cardoso SC, Primo RNG, Barros LFM. Segurança do paciente infantil no centro cirúrgico. Rev. SOBECC. 2013; 18(1): 44-53.

11. Brasil. Ministério da Saúde. Conselho Nacional de Saúde. Resolução 466/12. Pesquisas com seres humanos. Brasília: Ministério da Saúde; 2012.

12. Gomes AVO, Nascimento MAL, Christoffel MM, Antunes JCP, Araújo MC, Car$\operatorname{dim}$ MG. Punção venosa pediátrica: uma análise crítica a partir da experiência do cuidar em enfermagem. Enferm Global. 2011; 287-297.

13. Silva RNA. Validação clínica do diagnóstico "trauma vascular periférico" em crianças de 6 meses a 12 anos. [Dissertação Mestrado] Juiz de Fora: Faculdade de Enfermagem da Universidade Federal de Juiz de Fora; 2012.

14. Batalha LMC. Intervenções não farmacológicas no controle da dor em cuidados intensivos neonatais. Revista de Enfermagem Referência. 2010; III Série (2): 73-80.

15. Pedreira MLG, Peterlini MAS, Pettengill MAM. Ultra-sonografia na punção intravenosa periférica: inovando a prática de enfermagem para promover a segurança do paciente. Acta Paul Enferm. 2008; 21(4): 667-669.

16. Carneiro FS, Bezerra ALQ, Silva AEBC, Souza LP, Paranaguá TTB, Branquinho NCSS. Eventos Adversos na Clínica Cirúrgica de um Hospital Universitário: Instrumento de Avaliação da Qualidade. Rev enferm. UERJ. 2011; 19(2): 204-11.

17. Paranaguá TT, Bezerra AL, Silva AE, Azevedo Filho FM. Prevalência de incidentes sem dano e eventos adversos em uma clínica cirúrgica. Acta Paul Enferm. 2013; 26(3): 256-62.

18. Belela ASC, Peterlini MAS, Pedreira MLG. Revelação da ocorrência de erro de medicação em unidade de cuidados intensivos pediátricos. Rev Bras Ter Intensiva. 2010; 22(3): 257-263.

19. Sousa MRG, Silva AEBC. Análise dos eventos adversos decorrentes da assistência de enfermagem ocorridos em unidade de hemodiálise de um hospital de ensino da região Centro Oeste [Internet]. Anais do VI Congresso de Pesquisa Ensino e Extensão (CONPEEX); 27-30 outubro 2009. Goiânia: Universidede Federal de Goiás; 2009 [citado 01 agosto 2014]. Disponível em: http://www.ufg.br/conpeex/2009/.

20. Bohomol E, Ramos LH. Percepções sobre o erro de medicação: análise de respostas 
da equipe de enfermagem. Rev Lat Am Enfermagem. 2006; 14(6): 63-68.

21. Conselho Regional de Enfermagem. Erros de Medicação Definições e Estratégias de Prevenção [Internet]. São Paulo: Conselho Regional de Enfermagem; 2011 [citado 01 agosto 2014]. Disponível em http://intra.coren-sp.gov.br

22. Schatkoski AM, Wegner W, Algeri S, Pedro ENR. Segurança e proteção à criança hospitalizada. Rev Lat Am Enfermagem. 2009; 17(3).

23. Nascimento CCP, Toffoletto MC, Gonçalves LA, Freitas, WG, Padilha, KG. Indicadores de Resultados da assistência: Análise dos eventos adversos durante a internação hospitalar. Rev Lat Am Enfermagem. 2008; 16(4): 746-51.

24. Razmus I, Wilson D, Smith R, Newman E. Falls in Hospitalized Children. Pediatr Nurs. 2006; 32(6): 568-572.

25. Kissinger E, Marin A. Pediatric falls risk assessment in the hospitalized child. [Master's Thesis]. Sacramento: California State University; 2010. 52 p.

26. Conselho Regional de Medicina de São Paulo. Consulta No 23.606/97. Evasão de Pacientes de hospital psiquiatrico [Internet]. São Paulo: CREMESP; 1997 [citado 01 agosto 2014]. Disponível em: http:// www.cremesp.com.br/legislação/pareceres/parcrm/23606_1997.htm

27. Conselho Regional de Enfermagem de São Paulo. Parecer COREN-SP GEFIS N ${ }^{\circ}$ 27/2010. [Internet]. São Paulo: COREN; 2010 [citado 01 agosto 2014]. Disponível em: http://inter.coren-sp.gov.br/sites/default/files/027_2010_GEFIS_evasao_de_ pacientes.pdf.

28. Carmagnani MIS. Segurança e Controle de Infecção. Rio de Janeiro: Reichmann e Affonso Editous, 2002.
29. World Health Organization. World Alliance for Patient Safety. Forward Programme 2008-2009. Global Patient Safety Challenges [Internet]. Geneva: WHO 2008 [citado 01 agosto 2014]. Disponível em: http://www.who.int/patientsafety/ challenge/en/

30. World Health Organization. Guidelines on Hand Hygiene in Health Care [Internet]. First Global Patient Safety Challenge Clean Care is Safer Care. Geneva: WHO 2009 [citado 01 agosto 2014]. Disponível em: http://whqlibdoc.who. int/publications/2009/9789241597906 eng.pdf. Acesso: 08 de agosto de 2014.

31. Brasil. Ministério da Saúde. Agência Nacional de Vigilância Sanitária. Pediatria: prevenção e controle de infecção hospitalar - (Série A. Normas e Manuais Técnicos) Brasília: Ministério da Saúde, 2005. $116 \mathrm{p}$.

32. Silva RCL, Figueiredo NMA, Meireles IB. Feridas: fundamentos e atualizações em enfermagem. São Caetano do Sul, SP: Yendis, 2011.

33. Carvalho GB, Silva FAA, Castro ME, Florêncio RS. Epidemiologia e riscos associados a úlceras por pressão em crianças. Cogitare enferm. 2011; 16(4): 640646.

34. Blanes L, Duarte IS, Calil JA, Ferreira LM. Avaliação clínica e epidemiológica das úlceras por pressão em pacientes internados no hospital São Paulo. Rev Ass Med. Bras. 2004; 50(2): 182-187.

35. Freitas JS, Silva AEBC, Minamisava R, Bezerra ALQ, Sousa MRG. Qualidade dos cuidados de enfermagem e satisfação do paciente atendido em um hospital de ensino. Rev Lat Am Enfermagem. 2014; 22(3): 454-60. 